\title{
Biofabrication (3D Bioprinting) Laboratory at Sichuan University
}

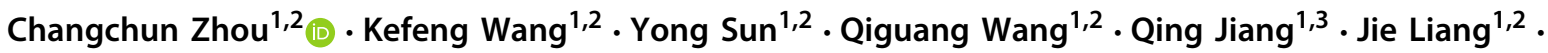 \\ Xuan $\mathrm{Pei}^{1,2} \cdot$ Boqing Zhang ${ }^{1,2} \cdot$ Yujiang Fan ${ }^{1,2} \cdot$ Xingdong Zhang ${ }^{1,2}$
}

Received: 26 October 2020 / Accepted: 17 November 2020 / Published online: 3 January 2021

(c) Zhejiang University Press 2021

\section{Introduction and research overview}

Recently, increasing need for organ transplantation and lack of donated organs have led to the rapid development of new technologies for artificial organ biofabrication. In the era of burgeoning breakthroughs around 3D bioprinting technologies, the personalization of organs and medicine is an ongoing nice vision [1-5]. As one of the leading laboratories in the interdisciplinary field of materials, manufacturing and bioengineering, the Biofabrication (3D Bioprinting) Research Laboratory at Sichuan University has been engaging in the research on customized regenerative medicine since 2012.

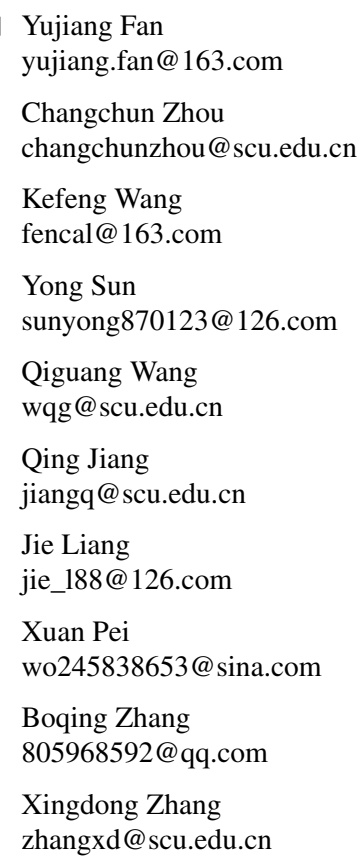

1 National Engineering Research Center for Biomaterials, Sichuan University, Chengdu 610064, China

2 College of Biomedical Engineering, Sichuan University, Chengdu 610064, China

3 College of Materials Science and Engineering, Sichuan University, Chengdu 610064, China
As a newly established research institute, the laboratory mostly focuses on regenerative biomedical engineering research, particularly on bone tissue-inducing biomaterials. Tissue-inducing biomaterial was officially defined as "a biomaterial designed to induce the regeneration of damaged or missing tissues or organs without the addition of cells and/or bioactive factors." The definition of tissue-inducing biomaterial was firstly proposed by Professor Xingdong Zhang, academic leader of our research center, and gained official consent at the congress on Definitions of Biomaterials for the Twenty-First Century, Elsevier, 2019. The orthopedic regenerative biomaterial fabricated by our research group is a successful tissue-inducing biomaterial belonging in this research field. It can be traced back to 1980s. Tissue-inducing biomaterial breaks through the traditional conception that biomaterials cannot induce the regeneration of tissues. Accordingly, it is our proposition that biofabrication or 3D bioprinting technology could be applied to the regeneration of personalized complex tissues.

\section{Involved printing materials}

The Biofabrication (3D Bioprinting) Research Laboratory at Sichuan University attempts to innovative researches and commercialization through the combination of engineering and biomedicine. The purpose of the laboratory is to promote the industrialization of technology and market operation, to meet the needs of personalized biomedicine and treatment to patients and to help to construct a harmonious and healthy society. Up to now, the researches of the laboratory involved 3D printing of bioceramics, metal, polymers and their composites. Some recent highlights are listed below.

\section{D printing of bioceramics}

3D printing of bioceramics, especially the bioactive calcium phosphate bioceramics, has drawn considerable attention in recent years [6-8]. Bioceramics possess exceptional bio- 


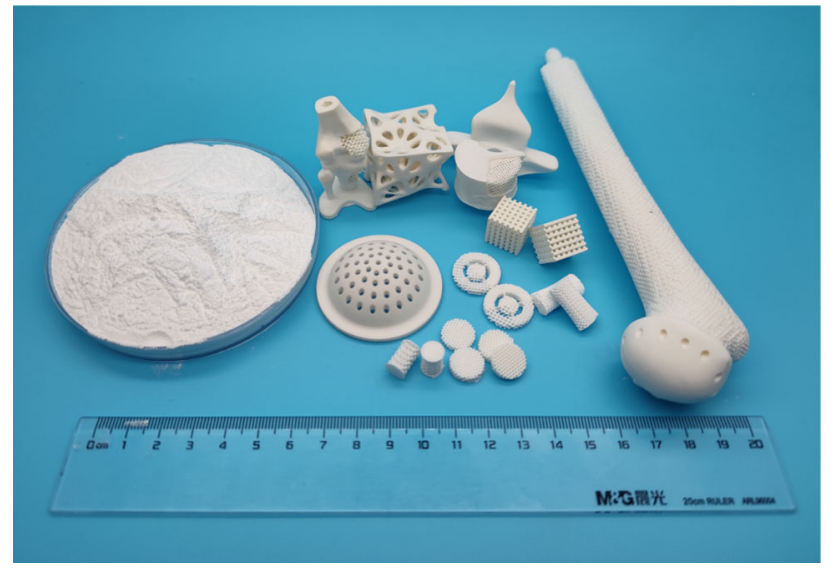

Fig. 1 Different 3D-printed calcium phosphate bioceramics. The customized 3D-printed calcium phosphate bioceramics can be used for maxillofacial bone repair, tumor bone defect filling, articular cup and elbow bone replacement

compatibility and bioactivity with respect to bone cells and tissues, due to their similarities to the chemical components and mineral structure of the bone tissues. The calcium phosphate bioceramic is beneficial to biomineralization in bone tissue regeneration. Up to now, bioceramics with different structures, shapes and biological functions can be successfully printed. Studies are being conducted on the applications of filling ceramic, cements, bearing bone substitute, component materials, or coating on orthopedic implants. Calcium phosphate powders (HA, $\beta$-TCP, BCP) formulated with different proportions can be used for printing ink configuration. The scaffolds may be fabricated by either inkjet or DLP 3D printing to obtain the porosity of $40-95 \%$. The optimal pore sizes ranging from $150 \mu \mathrm{m}$ to $800 \mu \mathrm{m}$ were recognized for bone tissue growth and reconstruction. It has been proved in our research that the 3D-printed calcium phosphate bioceramic shows excellent osteoconduction and osteoinduction; hence, it is a promising biomaterial for bone repair. Furthermore, the degradation rate of the bioceramic can be controlled by adjusting the porous structure and the material composition, which may tailor the biodegradation rate to match the growth rate of new bone regeneration [8]. Figure 1 shows different 3D-printed calcium phosphate bioceramics developed in our center, and some of them are successfully used in clinic. These special biofunctional ceramics show advantages in inducing bone tissue regeneration.

\section{D printing of metals}

The ideal scaffold for bone tissue reconstruction should resemble natural bones in both structural and mechanical properties. Owing to its excellent mechanical properties and biocompatibility, titanium alloy has been considered as the best candidate for 3D printing of bone tissue implants. Selec- tive laser melting (SLM) printer melts the selected area of titanium powder directly to manufacture an object layer by layer, so the object can be manufactured accurately $[9$, 10]. More importantly, SLM could produce highly complicated implants with customized architectures for different patients in accordance with their CT data. During modeling, with finite element analysis (FEA), the weakness of a structure under pressure can be simulated so that the structure can be predesigned and optimized. In our center, SLM technique was adopted to obtain precise porous titanium implants with pore sizes of 400-1000 $\mu \mathrm{m}$, exhibiting excellent osseointegration performance in vivo. Novel porous architectures, including cube structure, honeycomb structure and diamond-like structure, were designed for bionic fabrication of load-bearing bone scaffolds. According to studies, cortical bone exhibits elastic moduli and compressive strengths within the ranges of 7-20 GPa and 100-250 MPa, respectively, while in our study, the elastic modulus of the 3Dprinted scaffolds fabricated ranged from 1.19 to $5.14 \mathrm{GPa}$ and their compressive strength ranged from 36.76 to $139.97 \mathrm{MPa}$. They conform to the requirements for biomimetic mechanics. Studies have also been conducted on the removal of residual metal powders, heat treatment and surface biological activation of titanium alloy. It is found that the surface of the 3D-printed scaffolds can be further modulated by surface bioactivation to achieve favorable crystallinity and surface morphology. Figure 2 shows different 3D-printed porous titanium scaffolds for bone tissue engineering.

\section{D printing of polymers and composites}

Polymers can be easily printed and molded by various 3D printing approaches. Complex scaffolds can be produced according to 3D design files by decomposing an object's structures into a series of parallel slices. Then, the internal 3D structures are fabricated by reproducing these slices one layer at a time by using a sized nozzle. For photocured polymers, special photosensitive properties are required. Fused deposition modeling (FDM) technique is one of the most conventional and economical approaches; it is initially used to rapidly fabricate polymer products with geometric shapes and dimensions [11, 12]. FDM can be applied to print many different polymers, such as PLA, PS, PEEK and PCL. FDM technique has been adopted in fabricating polymer composites with different material components and customized geometries. Figure 3 shows different 3Dprinted polymers or polymer composites products prepared in our previous researches. Our center fabricated PLA/HA composites with enhanced osteogenic activity and mechanical properties. By combining the comprehensive optimized PLLA (L-polylactic acid)/nano-HA (nHA) composite with the low-cost FDM technology, PLLA/nHA porous bone tissue scaffold was achieved. The prepared PLLA/nHA 
Fig. 2 Different 3D-printed metal biomedical products. These 3D-printed medical-grade titanium or its alloys (Ti6Al4V) are fabricated into various biomimetic implants in terms of mechanical and structural properties, capable of being used in human orthopedics, such as femoral head nails or spinal fusion devices. (a, c, d) Femoral head nails and its porous structure units (b); (e) 3D printed Ti6Al4V spinal fusion products; (f) Samples of different microporous structure design; (g) Different designs of intervertebral fusion cage

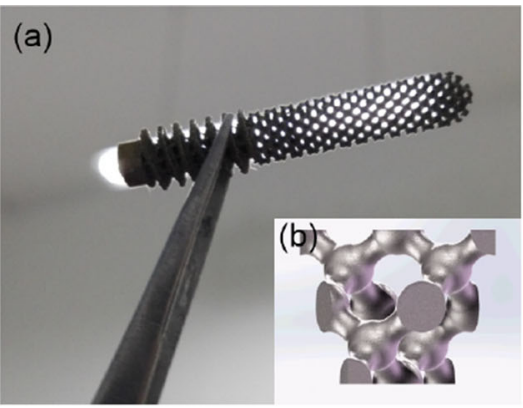

(c)
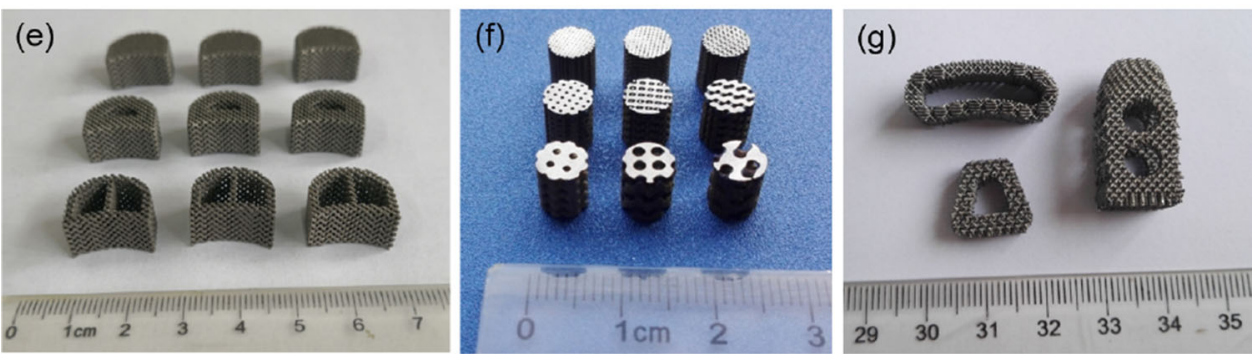

composite ink can secure the smooth and accurate printing required for personalized bone repair application. The PLLA/nHA composites scaffold has better mechanical properties and is free of the brittleness of porous bioceramics. At the same time, the scaffold has been proved with better osteogenic biological activity. PLA/HA composite scaffolds are more similar to natural bone tissues in terms of structure, composition and mechanical compatibility than those made of single ceramic or polymer materials. In addition, 3D bioprinting of cell-loaded polymers is another important research hot spot, capable of endowing the scaffold with a better biomimetic microenvironment and realizing precise assembly of tissue or organ cells in the spatial structure. It has been widely used in tissue regeneration and drug screening.

\section{Major research directions}

In the interdisciplinary field of 3D bioprinting, the main research applications/directions in our laboratory are mainly as follows:

\section{Bionic design and analysis of advanced regenerative biomaterials}

Tissue engineering involves the use of porous scaffolds to repair damaged biological tissues. The design and fabrication of porous scaffold still remain major challenges in bone tissue engineering. Hierarchical porous structures in scaffold endow tissue regeneration with different biological functions. Progresses in computational design and additive manufacturing (AM) have resulted in quick and accurate

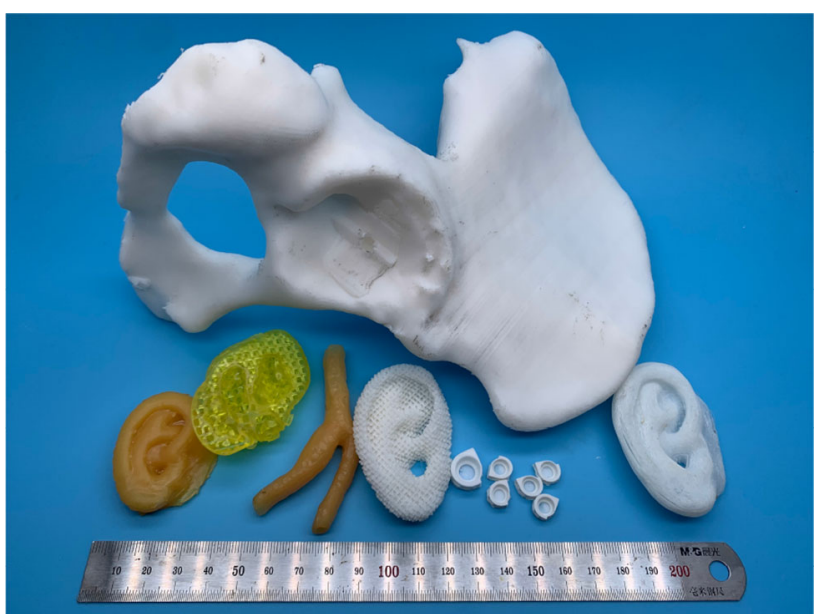

Fig. 3 Different 3D-printed polymers or polymer composites products. These polymer-based 3D-printed products have been studied for surgical models, surgical guide plates, artificial auricle stent, vascular stent, heart valve and so on. It shows good dimensional accuracy and mechanical properties

3D printing of porous scaffolds with well-controlled bionic architectures [13]. With thorough understanding of the structure of natural bone tissues, the personalized outer shape of the implant can be constructed according to a patient's personalized medical image data, such as CT and MRI. Based on the biomimetic natural bone trabeculae, a series of biomimetic architectures have been proposed, such as cube structure, honeycomb structure and diamond structure. With finite element analysis, the structures' weak points, optimal pore size and spatial distribution can be simulated so that the mechanical properties of the implants can be predesigned. Internal architectures of tissue implants can be filled with novel 3D porosity to achieve bionic design and manufac- 
turing. In addition, foaming techniques are combined with $3 \mathrm{D}$ printing for achieving higher resolution and higher efficiency of 3D printing. The potential and feasibility of these combined 3D printing technology open door to the creation of both macroscale porosity $(100-1000 \mu \mathrm{m}), 10-100 \mu \mathrm{m}$ and $1-10 \mu \mathrm{m}$ micropores in bionic porous scaffolds [14].

\section{Medical model and in vitro construction of diseased organs}

Various medical models and diseased tissues or organs have been fabricated by our research group. They could be used for doctor-patient communication, medical teaching, surgical planning and so on. Additionally, these models help to promote the design and preparation of customized prosthesis. Since the medical models do not need to have excellent biocompatibility as long as they can show personalized size characteristics, conventional printing technologies can be adopted, such as FDM and digital light procession (DLP). Models of bones, ears, tumor tissues, heart, blood vessels, etc., of specific patients can be well constructed in vitro. Light curing resin is a good choice to print material in this area (Fig. 4).

\section{Load-bearing bone tissue regeneration implants}

The repair of load-bearing bones and large defect bones is still a challenge in orthopedics clinic. At present, 3D printing technologies have been well tried [15, 16]. For example, clinical attempts have been made on the long spine, vertebral body, and femur substitutes. For these applications, biomechanical strength design, osseointegration with host bone tissue and the ability to reconstruct new bones are important issues need to be concerned. Studies have been conducted on 3D printing of metal-based implants loaded with active ingredients to promote bone integration, showing a good osseointegration effect in vivo. And it is expected to be used in spinal fusion cage.

\section{Craniomaxillofacial biodegradable osteoinduction implants}

Due to the diverse appearances of people, there are very highly personalized requirements for craniomaxillofacial bone tissue repair and reconstruction. 3D printing is a good choice for this kind of application. In our laboratory, personalized modeling has been studied based on patients' medical image data and fabrication of customized implants using biodegradable $\mathrm{CaP}$ bioceramics. With 3D printing of degradable calcium phosphate ceramics, the new bone tissue can be induced and the maxillofacial morphology can be well reconstructed, with the biological functions well repaired.

\section{Custom-made implants with specific biological functions}

With 3D printing, customized products can be flexibly composited and fabricated. By assembly of different components, or filling implants loaded with drugs, custom-made implants with specific biological functions can be fabricated. For example, adding bioactive ingredients into printing inks, or adsorbing drugs for controlled release has been tried to achieve various biological functions, like promotion of vascularization, anti-bacterial and anti-inflammatory functions $[17,18]$.

\section{In vitro biofabrication of live tissue engineering chips, organs and tissues}

Biofabrication is considered as a cutting-edge research in the emerging field of manufacture and biological systems. This research involves biomaterials, living cells, proteins and/or other biological compounds. They are used as basic building blocks for the fabrication of biomimetic structures, in vitro functional biological models and/or cellular systems applied to tissue engineering, regenerative medicine, disease pathogenesis, drug screening and tissue/organ chips. Biofabrication has great potential in drug screening, artificial organ construction and so on. This cutting-edge research is under way in our laboratories.

\section{Facilities}

Biofabrication (3D Bioprinting) Research Laboratory is affiliated to National Engineering Research Center for Biomedical Materials (NERB), Sichuan University. NERB is a professional biomedical materials research and development institution, which was founded by Ministry of Science and Technology of China in 2000 as the first open national biomedical materials research and development institution. It integrates academic research, industry and production of biomaterials or biomedical devices. The internationally reputed NERB processes first-class engineering research conditions and the innovation ability. The center was the first company in China to research and develop hydroxyapatite (HA) ceramics and related synthetic bone grafts/dental implants, and several biomedical products have been issued by National Medical Products Administration, NMPA (it was formerly known as the CFDA), with Registration Certificates for Medical Devices. More than 20,000 implants were successfully applied to patients. At present, we are promoting the customization of personalized medical products based on 3D printing technology. Biofabrication (3D Bioprinting) Research Laboratory has different types of laboratories for varied types of material printing, such as polymer 3D 
Fig. 4 Bionic design and analysis of advanced regenerative biomaterials. (a) Design of mandibular prosthesis assembly model (top) and products (below); (b) Pelvic bone repair products; (c, f, $\mathbf{g}$, i) Biomimetic modeling, design and mechanical strength simulation process of spinal fusion cage products, which enable the realization of pre-design analysis and surgical assembly; (d, e) Spinal microenvironment and mechanical simulation; (h) 3D printed spinal fusion cage
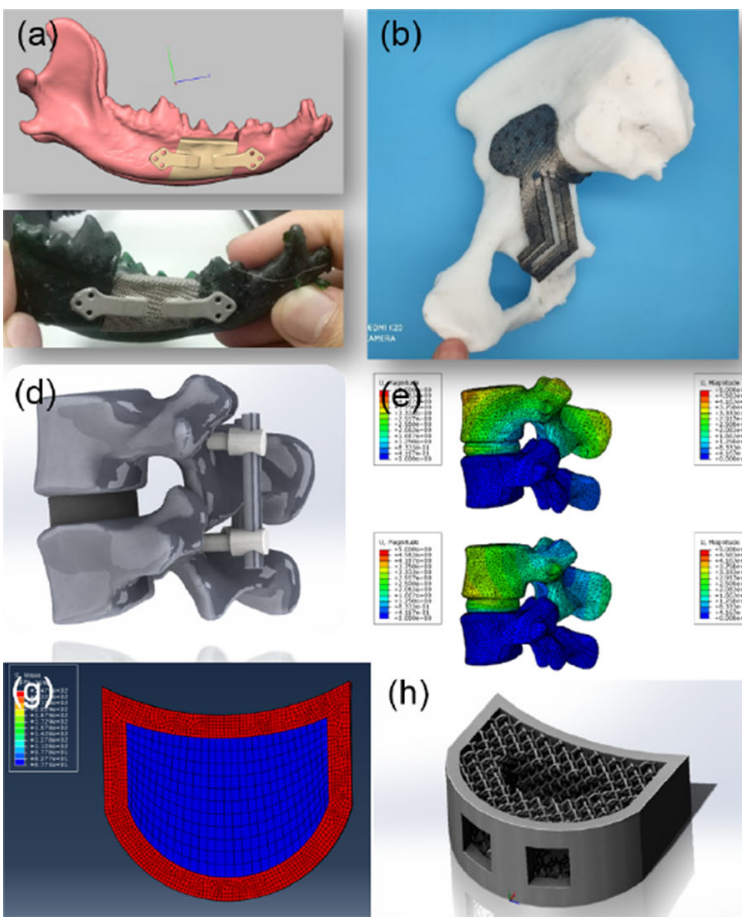

(h)

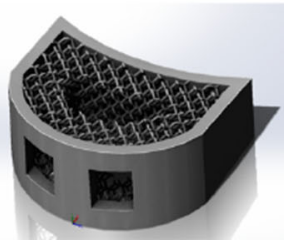

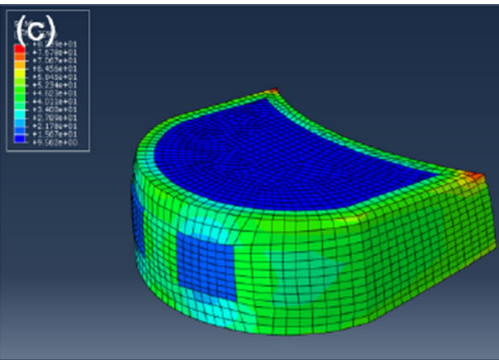

(f)

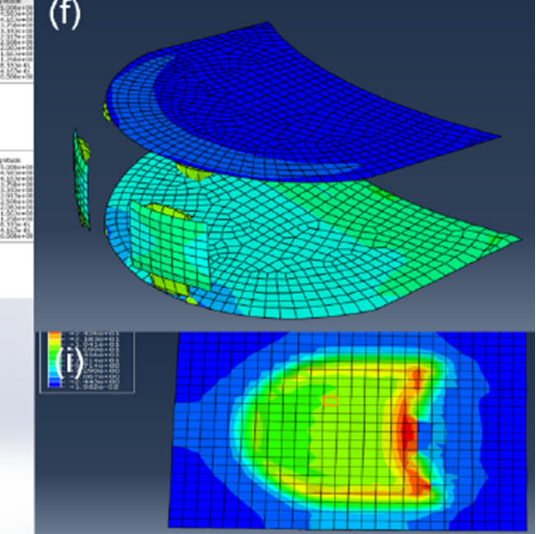

Fig. 5 Laboratories and research infrastructure of Sichuan University Biofabrication Research Laboratory. (a) Polymer 3D bioprinting room; (b) Ceramic 3D printing room; (c) Metel 3D printing room; (d) In vitro cell culture room; (e) 3D biofabrication lab
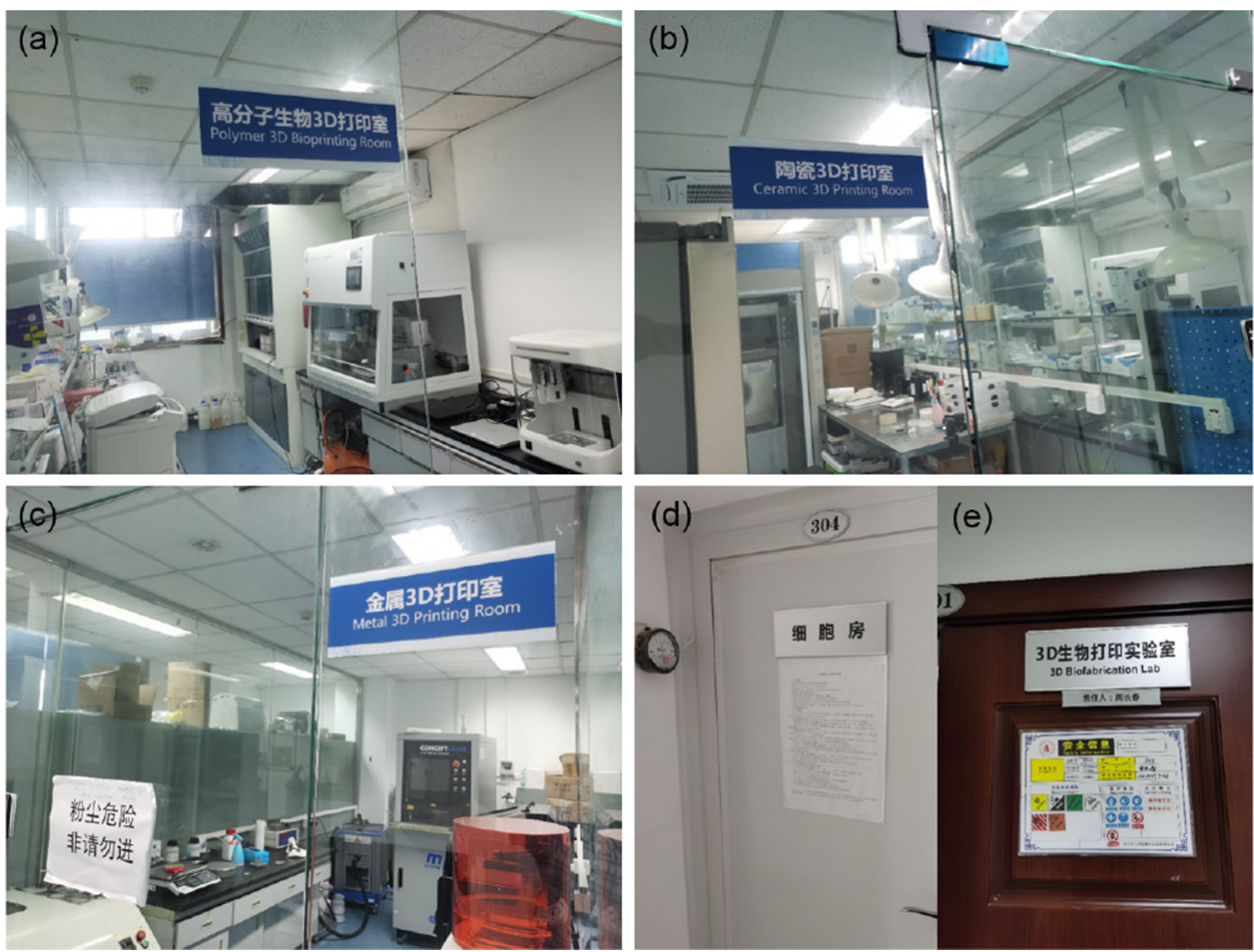

bioprinting laboratory, ceramic 3D printing laboratory and metal 3D printing laboratory (Fig. 5). These laboratories are equipped with different printing facilities to meet the needs of design and fabrication of different biomaterials. The 3D printer facilities include polymer material extruder, fused deposition modeling (FDM) printers, direct extrusion 3D printer, cardiovascular 3D printer, low-temperature deposi- tion printer, SLM metal 3D printer, bioceramics 3D printers, cell 3D bioprinter, photocuring 3D printer, etc. (Figure 6). These professional laboratories and research infrastructure meet the requirements for the materials' preparation, characterization, biological testing and evaluation, including in vitro cell experiments and in vivo animal experiments (Fig. 7). 

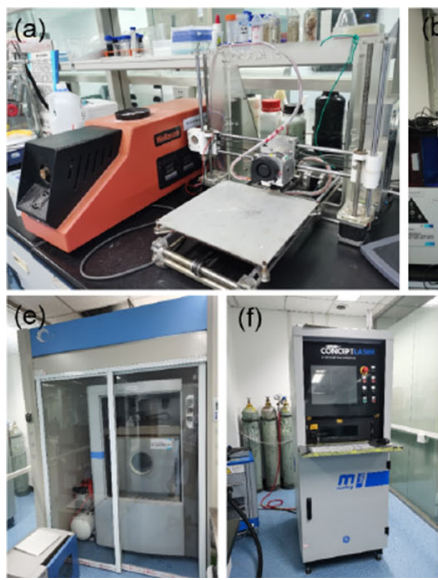

Fig. 6 Research infrastructure and 3D printers in Biofabrication Research Laboratory at Sichuan University. The 3D printing devices include: (a) polymer material extruder; (b) direct extrusion 3D printer, Regenovo; (c) fused deposition modeling, FDM printers; (d) cardiovas-
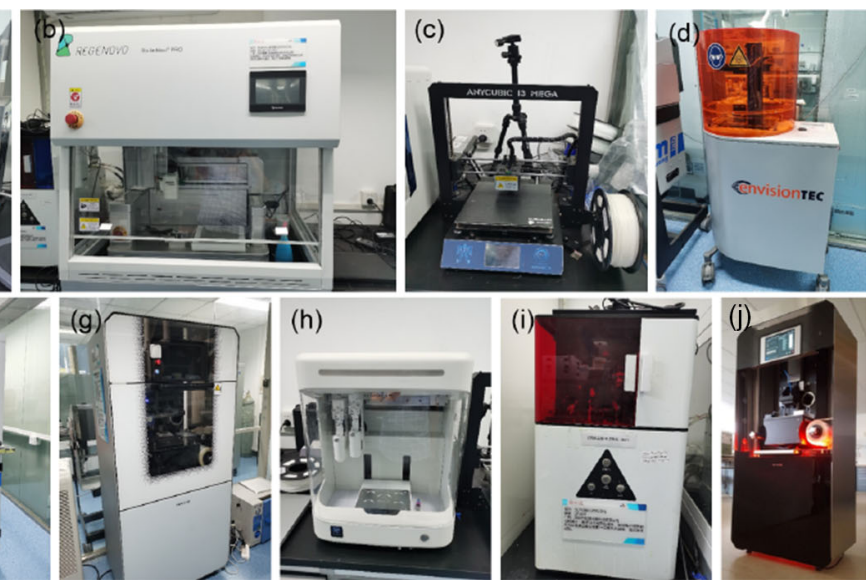

cular 3D printer: EnvisionTEC; (e) low-temperature deposition printer: SunP Biotech; (f) SLM metal 3D printer: Concept Laser; (g) 3D printer for bioceramics: ADMATEC; (h) cell 3D bioprinter: SunP Biomaker; (i) photocuring 3D printer; (j) DLP ceramics printer
Fig. 7 Laboratories and research infrastructure of Sichuan University Biofabrication Research Laboratory. The images in top row $(\mathbf{a}-\mathbf{c})$ are the in vitro cell culturing laboratories, and the bottom row images $(\mathbf{d}-\mathbf{g})$ are the animal experimental facilities
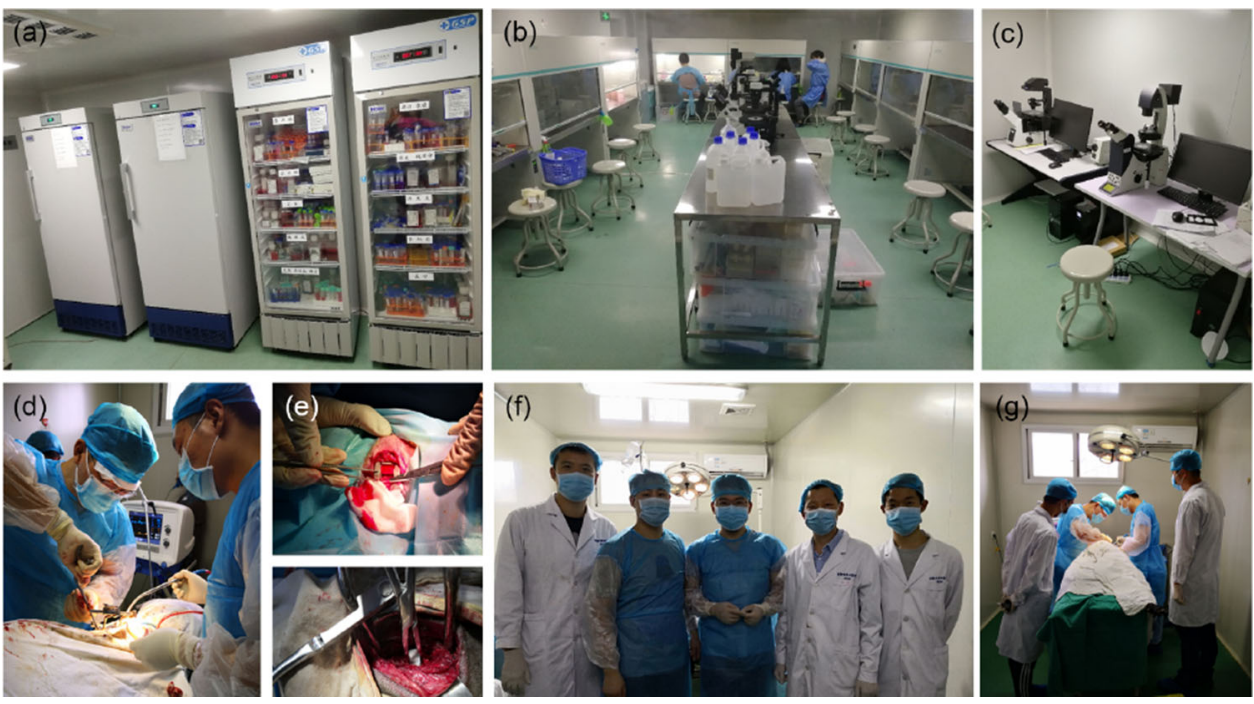

Fig. 8 (a) Group photographs of professor Xingdong Zhang, Academician of Chinese Academy of Engineering, and academic leader in our research center. (b) Group photographs of some PI professors and students participated in the $3 \mathrm{D}$ printing research
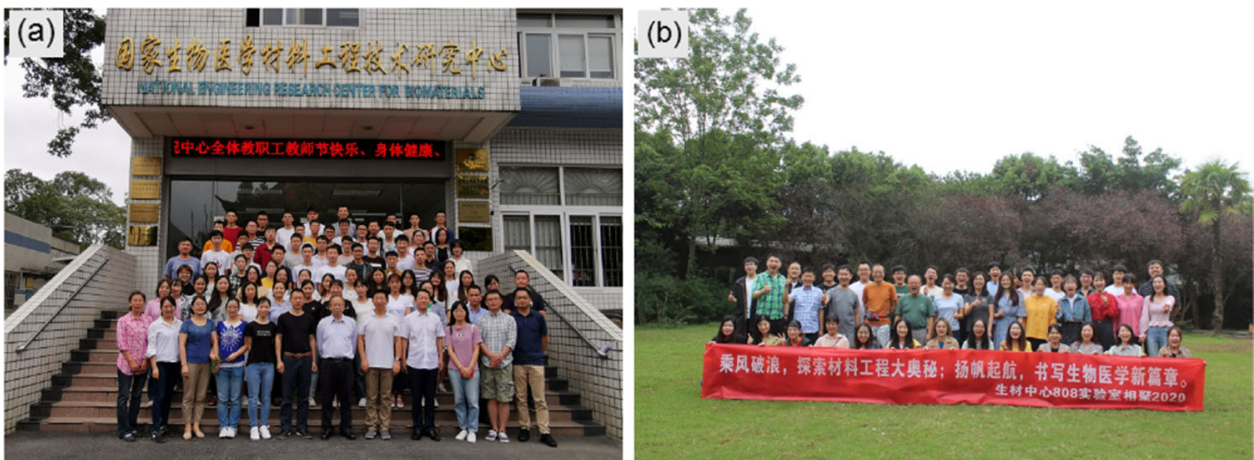

Combining the latest technologies in the field of 3D printing and clinical medical needs, the Biofabrication (3D Bioprinting) Research Laboratory is committed to improving patients' personalized medical plans and providing better health services. Figure 8 shows the recent group photographs of our research team, including some PI professors and students participating in the $3 \mathrm{D}$ printing researches. 
Our research work/projects have been sponsored by the National Key Research and Development Program of China, National Natural Science Foundation of China, Sichuan Province Science \& Technology Department Projects and H2020-MSCA-RISE of European Union. To this date, the center has published over 50 original research papers and applied for over 30 patents in the area of $3 \mathrm{D}$ printing or biofabrication of various novel biomaterials or biomedical devices. Some authorized patents have been successfully transformed into clinical production. Five patents belonging to Changchun Zhou and Yujiang Fan et al. were evaluated by a third evaluation party and priced at 16.9 million CNY. They jointly established Chengdu Bainian Beiya Medical Technology Co. Ltd. to promote personalized medical services for patients and better realize the transformation and application of 3D-printed orthopedic medical devices.

\section{Collaborations}

The center has undertaken a series of national research projects and enjoys extensive cooperation and exchanges with many domestic and overseas universities and research institutions in this field, including Tsinghua University, Zhejiang University, Shanghai Jiao Tong University, South China University of Technology, Xi'an Jiaotong University, Huazhong University of Science and Technology, Beihang University, Donghua University, Shanghai Institute of Ceramics, Chinese Academy of Sciences, Shenzhen institutes of advanced technology, Chinese Academy of Sciences, the European Society for Biomaterials, National Research Council, Italy, etc. Meanwhile, we maintain close cooperation with more than 20 domestic first-class hospitals, such as West China Hospital of Sichuan University, West China Hospital of Stomatology Sichuan University, and Southwest Hospital. We are collaborating in doing scientific researches or biomedical products clinical trials. Due to its good international influence and reputation, the Center has been designated as the Innovation International Talents Base (111 Base) by the Ministry of Education and the State Administration of Foreign Experts of China. More than 10 universities and research institutions worldwide in the field of biomaterials and 3D printing keep extensive international collaborations and exchanges with our center.

\section{Perspectives}

The Biofabrication (3D Bioprinting) Research Laboratory seeks to become a leading research center for medical scientific researches and technology innovation. We are dedicated to turning the center into a place well known for its merits in scientific researches, engineering and medical excellence.
And we are working to create customized biomedical products benefiting the living generations and the generations to come. As mentioned previously, our efforts are highly directed toward researches on orthopedic repair biomaterials, and plastic and cosmetic products. We are eager for further innovation and development in this field.

Author contributions $\mathrm{CZ}$ and $\mathrm{KW}$ contributed to methodology, investigation. YS, QW helped in writing and review. QJ, JL and XZ contributed to conceptualization and funding acquisition. XP, BZ contributed to original draft writing. YF contributed to supervision.

\section{Compliance with ethical standards}

Conflicts of interest There are no conflicts to declare.

Ethical approval The animal experiments were approved by the Animal Care and Use Committee of Sichuan University. All applicable international, national, and/or institutional guidelines for the care and use of animals were followed.

\section{References}

1. Ahadian S, Khademhosseini A (2018) A perspective on 3D bioprinting in tissue regeneration. Bio-Des Manuf 1:157-160. https:// doi.org/10.1007/s42242-018-0020-3

2. Li T, Zhou X, Deng C, Yang X, Yang H, Ma Z, Cao L, Yang Z, Qiang L, Wang J, Dai K (2019) Research center of 3D bioprinting in Shanghai Ninth People's Hospital. Bio-des Manuf 2:213-220. https://doi.org/10.1007/s42242-019-00045-8

3. Wang L, Li D, He J, Bingheng L (2018) Research center of biomanufacturing in Xi'an Jiaotong University. Bio-Des Manuf 1:280-288. https://doi.org/10.1007/s42242-018-0026-x

4. Zhang T, Lin F, Tao X, Zhang L, Yao R, Pang Y, Mi S, Ma S, Sun W (2019) Bio-Manufacturing Research Center at Tsinghua University. Bio-Des Manuf 2:137-143. https://doi.org/10.1007/s4 2242-019-00037-8

5. Zhang YS, Khademhosseini A (2020) Engineering in vitro human tissue models through bio-design and manufacturing. Bio-Des Manuf 3:155-159. https://doi.org/10.1007/s42242-020-00080-w

6. Pei X, Ma L, Zhang B, Sun J, Sun Y, Fan Y, Gou Z, Zhou C, Zhang X (2017) Creating hierarchical porosity hydroxyapatite scaffolds with osteoinduction by three-dimensional printing and microwave sintering. Biofabrication 9(4):045008-045020. https://doi.org/10. 1088/1758-5090/aa90ed

7. Zhang B, Pei X, Song P, Sun H, Li H, Fan Y, Jiang Q, Zhou C, Zhang $X$ (2018) Porous bioceramics produced by inkjet 3D printing: effect of printing ink formulation on the ceramic macro and micro porous architectures control. Compos B 155:112-121. https://doi.org/10. 1016/j.compositesb.2018.08.047

8. Zhang B, Sun H, Lina W, Ma L, Xing F, Kong Q, Fan Y, Zhou C, Zhang X (2019) 3D printing of calcium phosphate bioceramic with tailored biodegradation rate for skull bone tissue reconstruction. Bio-Des Manuf 2:161-171. https://doi.org/10.1007/s42242-019-0 0046-7

9. Pei X, Zhang B, Fan Y, Zhu X, Sun Y, Wang Q, Zhang X, Zhou C (2017) Bionic mechanical design of titanium bone tissue implants and 3D printing manufacture. Mater Lett 208:133-137. https://doi. org/10.1016/j.matlet.2017.04.128 
10. Zhang B, Pei X, Zhou C, Fan Y, Jiang Q, Ronca A, Ugo D'Amora Yu, Chen HL, Sun Y, Zhang X (2018) The biomimetic design and $3 \mathrm{D}$ printing of customized mechanical properties porous Ti6Al4V scaffold for load-bearing bone reconstruction. Mater Des 152(15):30-39. https://doi.org/10.1016/j.matdes.2018.04.065

11. Zhou C, Yang K, Wang K, Pei X, Dong Z, Hong Y, Zhang $X$ (2016) Combination of fused deposition modeling and gas foaming technique to fabricated hierarchical macro/microporous polymer scaffolds. Mater Des 109:415-424. https://doi.org/10.10 16/j.matdes.2016.07.094

12. Song P, Zhou C, Fan H, Zhang B, Pei X, Fan Y, Jiang Q, Bao R, Yang Q, Dong Z, Zhang X (2018) Novel 3D porous biocomposite scaffolds fabricated by fused deposition modeling and gas foaming combined technology. Compos Part B 152(1):151-159. https://doi. org/10.1016/j.compositesb.2018.06.029

13. Zhao L, Pei X, Jiang L, Cheng H, Sun J, Xing F, Zhou C, Fan Y, Zhang X (2019) Bionic design and 3D Printing of porous titanium alloy scaffolds for bone tissue repair. Compos B 162:154-161. https://doi.org/10.1016/j.compositesb.2018.10.094

14. Lina W, Zhou C, BoqingZhang HL, Wang W, Xiaobing P, Liu L, Liang J, Fan Y, Zhang X (2020) Construction of biomimetic natural wood hierarchical porous structures bioceramic with micro/nano whisker coating to modulate cellular behavior and osteoinductive activity. ACS Appl Mater Interfaces 12:48395-48407. https://doi. org/10.1021/acsami.0c15205
15. Song P, Cheng H, Pei X, Sun J, Sun H, Lina W, Jiang Q, Fan H, Yang B, Zhou C, Fan Y, Zhang X (2019) Dual modulation on crystallinity and macro/micro structures of $3 \mathrm{D}$ printed porous titanium implants to enhance the stability and osseointegration. J Mater Chem B 7:2865-2877. https://doi.org/10.1039/C9TB00093C

16. Yi T, Zhou C, Ma L, Lina W, Xiujuan X, Linxia G, Fan Y, Xian G, Fan H, Zhang X (2020) Direct 3D printing of Ti-6Al-4V/HA composite porous scaffolds for customized mechanical properties and biological functions. J Tissue Eng Regen Med 14:486-496. https://doi.org/10.1002/term.3013

17. Sun H, Cheng H, Zhou C, Lina W, Sun J, Zhou X, Xing F, Long C, Kong Q, Liang J, Fan Y, Zhang X (2020) 3D printing of calcium phosphate scaffolds with controlled release of antibacterial functions for jaw bone repair. Mater Des 189:108540-108553. https:// doi.org/10.1016/j.matdes.2020.108540

18. Pei X, Lina W, Zhou C, Fan H, Gou M, Li Z, Zhang B, Lei H, Sun H, Liang J, Jiang Q, Fan Y, Zhang X (2021) 3D printed titanium scaffolds with homogeneous diamond-like structures mimicking that of the osteocyte microenvironment and its bone regeneration study. Biofabrication 13:015008. https://doi.org/10.1088/1758-50 90/abc060 